\title{
The quality of rapid HIV testing in South Africa: an assessment of testers' compliance.
}

\author{
Aziza Mwisongo $^{1}$, Karl Peltzer ${ }^{1,2,3}$, Neo Mohlabane ${ }^{1}$, Bomkazi Tutshana ${ }^{1}$
}

1. HIV/AIDS/STIs/and TB (HAST), Human Sciences Research Council, Pretoria, Durban, South Africa.

2. Department of Research \& Innovation, University of Limpopo, Turfloop Campus, Private Bag X1106, Sovenga 0727, South Africa.

3. ASEAN Institute for Health Development, Madidol University, Salaya, Phutthamonthon, Nakhonpathom, Thailand, 73170 .

\begin{abstract}
Background: The aim of this study was to assess the quality of rapid HIV testing in South Africa.

Method: A two-stage sampling procedure was used to select HCT sites in eight provinces of South Africa. The study employed both semi-structured interviews with HIV testers and observation of testing sessions as a means of data collection. In total, 63 HCT sites (one HIV tester per site) were included in the survey assessing qualification, training, testing practices and attitudes towards rapid tests. Quantitative data was analysed using descriptive statistics and qualitative data was content analysed.

Results: Of the $63 \mathrm{HIV}$ testers, $20.6 \%$ had a nursing qualification, $14.3 \%$ were professional counsellors, $58.7 \%$ were lay HIV counsellors and testers and 6.4\% were from other professions. Most HIV testers (87.3\%) had had a formal training in testing, which ranged between 10-14 days, while $6(9.5 \%)$ had none. Findings revealed sub-standard practices in relation to testing. These were mainly related to non-adherence to testing algorithms, poor external quality control practices, poor handling and communication of discordant results.

Conclusion: Quality of HIV rapid testing may be highly compromised through poor adherence to guidelines as observed in our study.

Keywords: HIV rapid testing, quality, health workers, quality assurance

DOI: http://dx.doi.org/10.4314/ahs.v16i3.2

Cite as: Mwisongo A, Peltzer K, Moblabane N, Tutshana B. The quality of rapid HIV testing in South Africa: an assessment of testers' compliance. Afri Health Sci 2016;16(3): 646-654. DOI: http:// dx.doi.org/10.4314/abs.v16i3.2.
\end{abstract}

\section{Background}

HIV counselling and testing (HCT) is the gateway for referrals to key and important health care services related to prevention of mother to child transmission (PMTCT), sexually transmitted infections (STIs), anti-retroviral treatment (ART) and tuberculosis (TB). In the past, before the introduction of rapid HIV tests and reliance on laboratory tests, HIV testing was hindered by the long interval between 1-2 weeks from the testing and receipt of results. This highly contributed to poor clients' return of results and thus missing the opportunity for further linkages of services. ${ }^{1,2}$ Rapid tests were thus developed to increase the uptake of HIV testing in both clinical and

\section{Corresponding author: \\ Karl Peltzer, HIV/AIDS/STIs/and TB (HAST), Human Sciences Research Council, Pretoria, Durban, South Africa, Email: kpeltzer@hsrc.ac.za}

nonclinical settings, by providing same day HIV results performed by non-laboratory staff with an average of 10 minutes. ${ }^{1,3,4-6}$ Rapid tests, thus facilitate early HIV diagnosis with subsequent linkages to care for HIV infected persons. ${ }^{5}$ It has also made the delivery of HIV services in South Africa much easier and faster, contributing towards the achievement of National targets. ${ }^{12}$

South Africa has clear policies on HCT with specific guidelines on testing. ${ }^{11,19}$ Furthermore, the South African guide on rapid testing is intended to ensure; quality; detect and reduce errors; improve consistency between sites and contain costs. ${ }^{19}$ The National HCT Policy (2010) under section five recommends an HIV testing algorithm that begins with a screening test, followed by a confirmatory test in cases where the screening test is reactive. It also stipulates that positive cases need to be referred for CD4 count, TB screening and pre-ART, while those who are non-reactive should be provided with counselling and encouraged to repeat the test after 3 months with a provision 
of an appropriate prevention package. ${ }^{11}$ For the accuracy and reliability of HIV rapid testing South Africa applies a quality system approach. ${ }^{19}$ The South African guideline stipulates that each testing site should have the following: proper organisation and management for HIV rapid testing; accuracy and reliability process control guide; finger prick blood collection guide; country approved testing strategy and algorithm; guidance to result interpretation; client result and quality data recording tools and client result reporting. ${ }^{19}$ The national, provincial, testing sites and the National Reference Laboratory, in this case the $\mathrm{Na}$ tional Institute for Communicable Diseases (NICD) all play a key role in managing rapid testing in South Africa. ${ }^{?}$

The guide stresses on the competencies of individual testers with emphasis on initial, ongoing training for Quality System (QS) and Quality Assurance (QA) and periodic evaluation of their testing skills. ${ }^{9}$ Tester's proficiency and competence are important as illustrated by research, for example a study from South Africa showed that the sensitivity and specificity of rapid tests performed by nurses and counsellors were $92.5-97.3 \%$ and $97.6-98.2 \%$, respectively, while laboratory technicians had a $100 \%$ performance. $^{7}$ The authors of the study believed that inadequate training of nurses and counsellors may have potentially caused such discrepancies. ${ }^{7}$ These results were, however, inconsistent with another study in Cambodia, where the accuracy of reports by VCT counsellors' scored $100 \%$, which was higher than laboratory technicians who scored $99.3 \% .^{10}$ In this study, human error in recording results was considered to be a potential reason for the discrepancy. ${ }^{10}$ The proficiency of personnel administering HIV testing has an impact on the sensitivity (ability of the kit to correctly detect specimens containing HIV antibodies) and specificity (ability of the kit to correctly detect specimens that do not contain HIV antibodies) of the test kits thus sufficient training and performance assessment is required. ${ }^{7,9}$

The process of testing is mainly concerned with correct testing procedures, environment and test kits for accurate results. Practically, this includes the use of pre-evaluated kits, monitoring, storage, Internal Quality Control (IQC), External Quality Control (EQA) with management and supervision. ${ }^{9}$ In addition, testing personnel are required to follow the manufacturer's instructions on the test kit and site specific procedures as part of the quality assur- ance program. ${ }^{9}$ In South Africa, the recommended HIV testing algorithm is the serial algorithm where specimens are tested by a highly sensitive test. ${ }^{12}$ In the serial approach, an HIV negative test is considered as a true negative and a client is required to come back in 3 months while an HIV positive result is confirmed using a different rapid test kit. ${ }^{9,12}$ Adherence to a laboratory protocol, storage of testing kits, expiry date and integrity of test kits, specimen collection, testing area temperature, test performance and results interpretation are important considerations for quality assurance in the testing process as revealed in other settings., ${ }^{5,12}$ Recording is important to the testing process and the Department of Health of South African insists on records of; client results; quality control results, temperature charts, stock card, EQA report and report of corrective actions. ${ }^{?}$

The quality assurance cycle emphasises on four main components; personnel competency and test evaluations, pre-test preparations/client preparation and sample collection, quality control and testing and record keeping and reporting. Several studies have pinpointed to the existing malpractices which have led to either false positive and negative HIV cases which in turn have adverse effects on an individual and on programmatic levels. However there are no studies that have assessed how these processes are adhered to by testers in South Africa. The aim of this study was to assess the quality of rapid HIV testing in South Africa.

\section{Methods}

\section{Study setting and design}

This analysis is based on data from an assessment of HCT sites that was conducted in eight provinces of South Africa in 2012. The study was conducted in both public and private-sector health facilities in order to document the spectrum of approaches to providing HCT services in South Africa. The study population included HCT service providers who interact directly with clients at a service delivery site level, including those who provide HIV counselling and/or testing. All the 63 HCT sites were assessed by a site visit, a number of research methods, both qualitative and quantitative were used to collect data. This paper draws findings from both the semi-structured interviews with an HIV tester at the HCT sites and the observation checklist of the counselling and testing sessions.

African Health Sciences Vol 16 Issue 3, September 2016 


\section{Sampling and procedures}

A two-stage sampling procedure was used. Firstly, one health district in each of the eight provinces in South Africa was randomly chosen for inclusion in the study for the public HCT sites. Secondly, from the available lists of public and private HCT sites four public and three private HCT sites were purposively sampled per health district. Public, private and non-governmental organisations (NGOs) were selected on the basis of service provision, e.g. HCT service in a specific setting, using a specific model/approach, and/or providing services to a specific target population. An effort was made to include examples of Provider Initiated Counselling and Testing (PICT), community-based HCT with community outreach, mobile HCT, couples HCT, and HCT as part of a workplace-based health service; sites targeting specific high-risk populations (e.g., Men who have sex with men (MSM), commercial sex workers (CSWs), drug users, youth); and HCT integrated with other relevant services, e.g. sexual transmitted infection (STI) clinics.

A semi-structured interview guide containing both closed and open-ended questions was used to collect information pertaining to experiences and practices of HIV testing. All interviews took place in a private setting after written informed consent was obtained from all respondents. A senior researcher administered the semi-structured questionnaire and recorded in writing all open-ended responses verbatim.

A checklist which contained information on HIV testing was assessed at each of the HCT sites whereby two to three pre and post-test counselling sessions were observed at each site. This section had three broad areas of observation; before testing; during testing and after testing. The main areas of observation were related to; provision of information; safety and biohazard precautions; application of algorithms; time used and recording. Observations were conducted by both the researcher and a trained research assistant. Written informed consent was obtained from both the counsellor and the client before observing a counselling session. Consent was also requested to make an audio-recording of the counselling session to assist the observer with capturing all items listed on the counselling assessment form, based on South African guidelines. ${ }^{11}$
Data collection was done by senior researchers AM, NM and BT, while KP provided the overall guidance and conceptualisation of the study. The questionnaire was mainly administered in English, Afrikaans, Zulu/Tswana or Xhosa. The study was approved by the ethics committee of the Human Sciences Research Council (Protocol REC 9/19/08/09; FWA number: 00006347 IRB number: 0000 3962) with approvals from Centres for Disease Control, National Department of health, and Departments of health from the eight study provinces.

\section{Data analysis}

All quantitative data were analysed using IBM SPSS for Windows version 20.0 (SPSS, Inc., Chicago, IL, USA). Descriptive statistics were used to describe characteristics of the testers. Differences between groups were examined using Pearson chi-square or Fisher's exact test. All open-ended responses were manually compiled. Firstly, all qualitative responses were read several times to get an overall picture and then meaningful units were coded, condensed and categorized into broad themes and through the coding of relevant responses, data interpretation and conclusions were drawn.

\section{Results}

\section{Characteristics of HIV testers}

Of the 73 HCT sites approached for inclusion in the study, 67 agreed and 6 refused (all NGOs), the response rate was $83.6 \%$. However, only 63 HCT sites had an HIV tester that was included in the study, since four of the sites did not have an HIV tester, as HIV testing was performed off site, usually in designated laboratories. The selected HCT sites were spread over eight of nine provinces, as permission to conduct the study could not be obtained from the provincial government of the North West province. Most of the HIV testers (92.1\%) worked on a general HCT site not having any specific target group such as men or farmers. The majority $(79.4 \%)$ of the HIV testers was based in fixed HCT sites, and two-thirds $(67.2 \%)$ worked in government health services, $29.5 \%$ in non-governmental organisations (NGOs), 1 (1.6\%) from private facility and $1(1.7 \%)$ from workplace based site. Private and workplace facilities had very few staff compared to NGOs and government facilities so that only two could be recruited from these sites (see Table 1). 
Table 1: Characteristics of the HIV testing sites where HIV testers were recruited $(\mathbf{n}=63)$

\begin{tabular}{|l|l|l|}
\hline Variables & $\mathbf{n}$ & $\mathbf{\%}$ \\
\hline HCT Type (target groups) & & \\
Specific group (men) & 4 & 6.4 \\
No specific group & 58 & 92.1 \\
Specific group (farmers) & 1 & 1.6 \\
\hline HCT type (fixed or mobile) & 50 & \\
Fixed & 5 & 79.4 \\
Mobile & 56 & 7.9 \\
Fixed and mobile & 2 & 9.5 \\
Mobile (campaigns) & & 3.2 \\
\hline Type of organization & 41 & 67.2 \\
Government health service & 18 & 29.5 \\
NGO & 1 & 1.6 \\
Workplace & 1 & 1.6 \\
Private health service & & \\
\hline HCT Type & 13 & 21.3 \\
Hospital & 27 & 44.3 \\
Primary health care clinic & 8 & 13.1 \\
Stand-alone (fixed) & 1 & 1.6 \\
STI clinics & 3 & 4.9 \\
Mobile HCT service & 4 & 6.6 \\
Outreach (e.g. at events) & 4 & 6.6 \\
Community Centre & 1 & 1.6 \\
Workplace & & \\
\hline Type of area & 14 & 23.0 \\
Urban & 15 & 24.6 \\
Peri-urban or townships & 3 & 4.9 \\
Informal settlements & 29 & 47.5 \\
Rural areas & & \\
\hline Province & 8 & 12.7 \\
Western Cape & 11 & 17.5 \\
Eastern Cape & 5 & 11.1 \\
Northern Cape & 7 & 7.9 \\
Free State & 8 & 11.1 \\
Kwa-Zulu Natal & 9 & 12.7 \\
Gauteng & 8 & 14.3 \\
Mpumalanga & & 12.7 \\
Limpopo & \\
\hline
\end{tabular}

Most HIV testers $(88.9 \%)$ were women. The median age was 38 years $(\mathrm{SD}=10.4)$, ranging from 19 to 71 years. Over half $(52.4 \%)$ of the HIV testers had a Grade 12 education, $14.3 \%$ had a post-secondary education and $33.3 \%$ had an education lower than Grade 12. Regarding the professional background, $20.6 \%$ were in nursing profession, $14.3 \%$ were professional counsellors, $58.7 \%$ were lay HIV counsellors and testers and 6.4\% were from other professions. The majority of HIV testers $(74 \%)$ had an experience in HIV testing of between a few months and five years, followed by $14(22.2 \%)$ between 5 and 10 years and $2(3.2 \%)$ with more than 10 years of experience in HIV testing. Most HIV testers (87.3\%) had had a formal training in testing, which ranged between 10-14 days, while $6(9.5 \%)$ had none. Four among the six HIV testers who had no formal training on testing were from non-governmental organisations (NGOs). A range of organisations were mentioned as a provider of HCT training, including NGOs, universities, department of health at either national, provincial or district level.

\section{Type of test kits}

Rapid tests were commonly used for both screening and confirmation tests, however, there were differences between the tests that were used for screening and for confirmation. The commonest rapid tests that were mentioned include; SD Bioline, First response, Advance quality, Determine and G-Ocean. Table 2 below shows the common screening and confirmatory tests used in the 8 studied provinces. Other uncommon rapid tests that were 
also mentioned were; Medmira rapid HIV tests and Abon rapid tests. These were mentioned by NGOs who had decided to replace them with existing tests due to unreliability. A few of the NGOs also mentioned the use of ELISA as a confirmatory test after screening with a rapid test. There were also a few sites that only did one test due to stock out or problems with the screening test such as Advance quality and G-Ocean. There weren't any differences between cadres of counsellors and their overall satisfaction of rapid HIV test.

\section{Table 2: Names of rapid tests commonly used for screening and confirmatory tests in study sites in 8 provinces of South Africa}

\begin{tabular}{|l|l|l|}
\hline Provinces & Screening tests & Confirmatory test \\
\hline Gauteng & Advance Quality & First response \\
\hline Free State & G-Ocean & Determine \\
\hline Eastern Cape & G-Ocean & First Response \\
\hline Northern Cape & First Response & Determine \\
\hline KwaZulu Natal & G-Ocean & Determine \\
\hline Limpopo & Advanced Quality & First response \\
\hline Mpumalanga & Advanced Quality & First response \\
\hline
\end{tabular}

\section{Testing proficiency}

When testers were asked about the HIV testing algorithm that they applied, $59(93.7 \%)$ of all testers declared to begin with a screening test, but not all of the testers 56 $(88 \%)$ continued to do a confirmatory test. Lay testers were significantly $(\mathrm{P}<0.001)$ more likely to conduct both screening and confirmatory tests compared to other cadres such as nurses and lab technicians. According to the testers, only $27(42.9 \%)$ conduct an additional test if there is a tie breaker between the screening and confirmatory tests, while the rest turn to other means such as telling the patient to come back after a few weeks or to provide them with an indefinite result. When testers were asked what influenced the decision of this algorithm, there were several responses: instructions from higher authority, availability of stocks, training and depending on test results (see Table 3).

Table 3: Reasons for choices of HIV testing algorithm

\begin{tabular}{|c|c|}
\hline Category of reasons & Reasons for choices of testing algorithm \\
\hline Higher authority guidance & $\begin{array}{c}\text { Instructions from higher authority } \\
\qquad \quad \text { Memos from the district management } \\
\bullet \quad \text { District guidance/guidelines } \\
\bullet \quad \text { Directives from the Department of Health }\end{array}$ \\
\hline Availability of stocks & $\begin{array}{l}\text { Availability/Type of stocks } \\
\qquad \quad \text { Availability of one test only } \\
\bullet \quad \text { Instruction by in charges } \\
\text { - Problems with some rapid tests, e.g. SD Bioline and } \\
\text { Advance Quality }\end{array}$ \\
\hline Training & $\begin{array}{l}\text { - Influence of service provider e.g. NGOs supporting } \\
\text { PHCs on counselling } \\
\text { - Influence of training }\end{array}$ \\
\hline Test results & $\begin{array}{c}\text { Influenced by results } \\
\bullet \quad \text { When results are discordant } \\
\bullet \quad \text { When results are not clear }\end{array}$ \\
\hline
\end{tabular}


From the observation checklist it was found out that the majority of testers follows the guidelines in terms of preparing for an HIV test. Among the testers 137 (97\%) provided test information to their clients before doing the actual procedure whilst 138 (98\%) set up the test area, $122(87 \%)$ labelled the testing devices and 141 (95\%) prepared the pricking needles. 94\%, followed Biohazard safety precautions.

Ninety-eight percent of the HIV testers that had been observed, collected a blood specimen for the screening test, while only $18 \%$ collected a specimen for the confir- matory test. Seventeen percent of the HIV testers collected the specimen once and divided it for both tests, $12 \%$ applied a parallel testing approach, and $70 \%$ applied a serial testing approach. Among the testers $67 \%$ documented the results, $99 \%$ reported the results to the client and $49 \%$ issued a receipt of the results to the client.

\section{Handling of indeterminate results and quality con- trol}

When testers were asked how they handle the situation when the results were discordant, testers mentioned the following procedures.

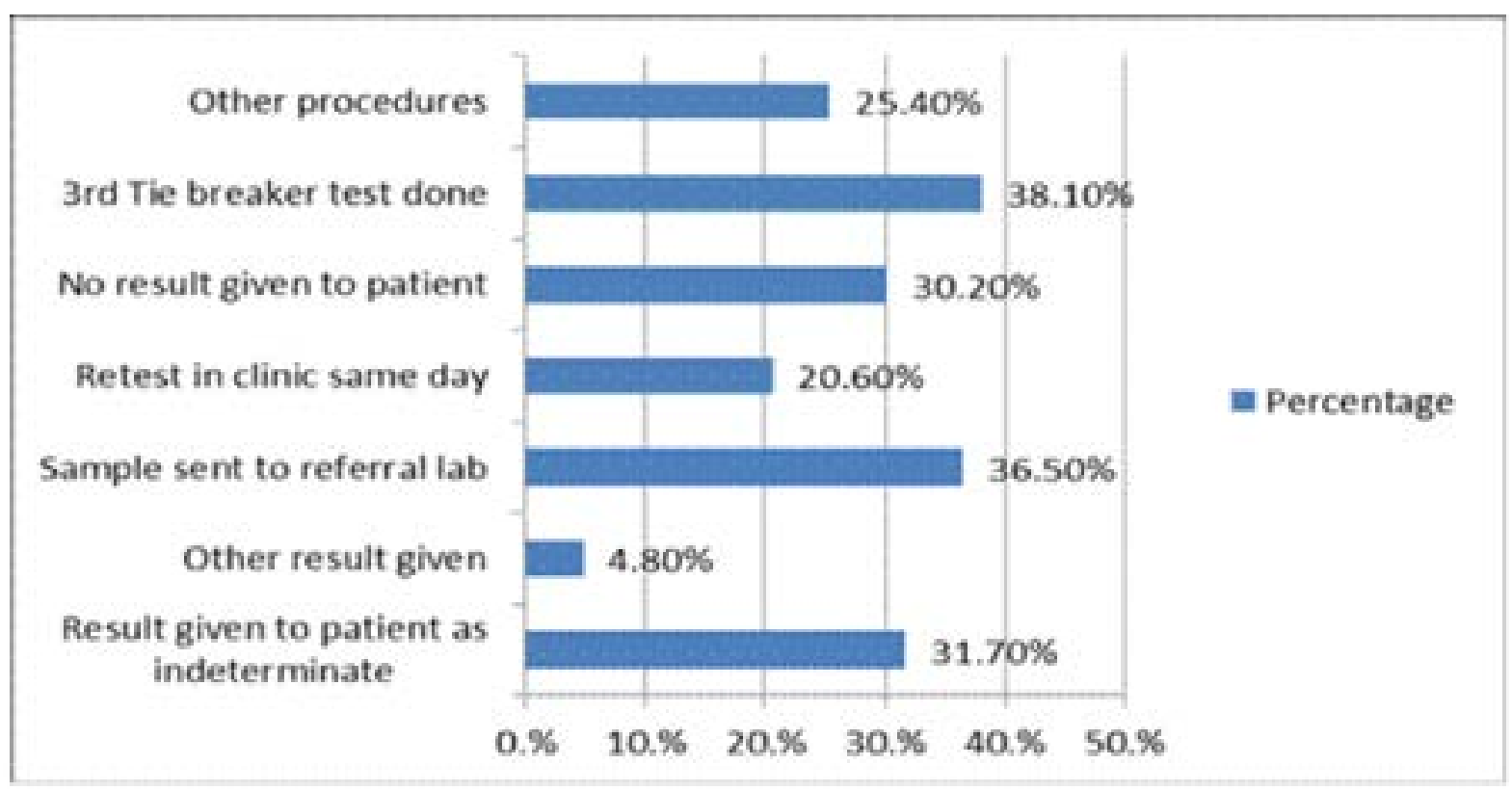

Figure 1: Procedures for handling indeterminate HIV test results by testers

Other responses included; referral to other clinics or and consultation of seniors to make a decision. From the qualitative responses in the semi-structured interview there was a lot of variation on how testers explained discordant results to patients. Some opted to explain that they could not figure out the results, others used lay language to explain to patients, but with no reasons and others just advise patients to return after some time for a repeat test. Majority of testers (both lay counsellors and nurses) commonly mentioned the comparison of every 5th test kit with an Eliza test as an external control mechanism for ensuring quality of HIV rapid tests. Furthermore, there was a tendency for the testers to trust the test kits and therefore did not constantly check and assure that test kits were actually accurate.

\section{Storage and stacks of HIV tests}

Among the testers, $32(61.5 \%)$ had access to a refrigerator at their sites for storage of blood and serum specimens. The rest who did not have a refrigerator made use of a cooler box, or transferring specimens to the lab on the same day and others claimed that they did not draw any blood and usually referred patients to the labs. Among the testers 59\% agreed to have a Standard Operating Procedures (SOPs) for handling an HIV test and its procedures, while $39.1 \%$ claimed not to have any and $1.6 \%$ did not know. However, this information highly contradicts with the actual presence of SOPs through observation, where only $5(16 \%)$ of the visited sites had any specific guidelines / protocol for HIV rapid testing while the rest had none.

African Health Sciences Vol 16 Issue 3, September 2016 
At least $51(85.5 \%)$ of all testers had the required test kits on the day of the assessment compared to $9(14.3 \%)$ who had a stock out. However, 34 (54\%) testers affirmed to experience a stock out of testing kits. The number of days of stock outs ranged between a few hours to 91 days.

\section{Discussion}

HIV rapid testing is one of the most cost-effective intervention, however, ensuring quality control is a challenge, especially in resource constrained contexts. ${ }^{13,18}$ In Low and Middle Income Countries (LMIC) existing challenges of Human Resources Health (HRH), mainly characterised by limitations in numbers and qualifications makes it even more complicated. ${ }^{13}$ It is evident from our study that lay testers play an important role in the provision of rapid testing that forms part of the HCT process. However, given their qualification which is generally low (Grade 12 and below) the conduct of such tasks can be challenging. It is also evident from other studies elsewhere that there exists a number of practices that raises doubt about the quality of rapid HIV testing in sub-Saharan Africa. ${ }^{14,15}$

Shanks et al. ${ }^{14}$ implemented a number of interventions in Burundi, Democratic Republic of Congo (DRC) and Ethiopia after they found a number of false positive results. From our assessment, there were some core areas that testers did not follow the stipulated guidelines of rapid HIV testing in South Africa. A common negligence was the performance of only one test with no follow up on a confirmatory test. This is a disturbing finding since evidence elsewhere has shown that even with the performance of two positive rapid HIV test there is a 10.5\% false positive rate. ${ }^{15}$ From a scientific justification two test algorithm is advised where a very sensitive assay is used as a screening and followed up with a more specific assay as a confirmatory test. ${ }^{19}$ The consequences of a false result are detrimental at both personal, system and programmatic level as stated by Klarkowski et al. ${ }^{15}$

From our study a number of factors were attributed to this practice such as lack of stock of tests for a confirmatory test, problems with either the recommended screening or confirmatory tests obliging testers to rely on one test and in other instances testers claimed to follow recommendations from higher authorities either at the site, NGO or districts/provinces, while other authors have mainly argued that the reason for this malpractice is due to variation in qualifications of health workers who actually perform the tests. ${ }^{13,18}$ Further, quality assurance of tests which is prudent was rarely practiced among testers in our assessment. This is a worrying practice since evidence has shown field examination of the sensitivity and specificity of rapid tests that are commonly used in South Africa is lower as compared to what the manufacturer's claim ${ }^{7}$ Dewsnap and Mcowan ${ }^{20}$ pointed out that the different rapid tests that were assessed by WHO differed immensely by; sensitivity, specificity, ease of performance, preparation time, storage, etc. which all need to be monitored on a routine manner as emphasised by the South African and other guidelines on rapid test. ${ }^{21}$

The fourth form of variance between the guidelines and practice the improper explanation of information to patients in relation to discordant results. In line with this was also poor follow up confirmatory actions required for discordant results. In our assessment, we found out that some of the testers were unable to explain or comprehend such discordant results and as a result, some of the testers did not follow up with an ELISA test for confirmation as per recommendation of the national HCT policy or provided false information to the clients. Additionally, the South African National HCT policy emphasises the need of follow up tests after three months for all negative patients, yet this was not communicated to clients by their testers. Among the positive-reactive clients, one common recommendation was for a follow up CD 4 count and many cases were not referred for TB screening. This is also supported from findings during the counselling session which were characterised by limited discussion related to Tuberculosis and other opportunistic infections by the counsellors.

\section{Study limitations}

There was limited sampling of the private sector. It was also difficult to select certain types of HCT sites due to a lack of comprehensive list of HCT sites in the country. Caution should be taken when interpreting these results because of the study limitations. The study sample included in the majority lay counsellors and testers and also professional nurses, so findings cannot be generalized for the experiences of all lay counsellors in the country. Another limitation relates to the reliability and validity of the questionnaires which were adopted from previous questionnaires and were extensively changed in line with the 
South African context. The assessment of training is also limited as the researchers did not make reference to the counsellor's minimum standard in determining if counsellors were properly trained. Lastly, since the study was not intended to assess task shifting, it is limited to make any conclusions about its execution.

\section{Conclusion}

Quality of HIV rapid testing may be highly compromised through poor adherence to guidelines as observed in our study. There is an urgent need for authorities in South Africa to intervene with the implementation of a Quality Management System, entailing; organisations and management; personnel; purchasing and inventory; process control; equipment and stocks and monitoring and evaluation. ${ }^{16,17}$ In particular, the strengthening of the quality systems should also take into consideration; training of HIV testing, testing approaches and algorithms, standardisation of testing kits, quality assurance (internal and external) and monitoring and evaluation as stipulated by World Health Organization guidelines. ${ }^{16,17}$

\section{Competing interests}

The author(s) declare that they have no competing interests.

\section{Author's contributions}

AM, KP and NM were the main contributors to the conceptualization of the study. AM, KP and NM contributed significantly to the first draft of the paper and all authors contributed to the subsequent drafts and finalization. All authors read and approved the final manuscript.

\section{Acknowledgements}

This research has been supported by the President's Emergency Plan for AIDS Relief (PEPFAR) through the Centers for Disease Control and Prevention (CDC) under the terms of Cooperative Agreement Number U2G/ PS000570.

\section{References}

1. Hutchinson A, Branson B, Kim A, Farnham, FG. A meta-analysis of the effectiveness of alternative HIV counselling and testing methods to increase knowledge of HIV status. AIDS 2006; 20:1597-1604

2. Sullivan PS, Lansky A, Drake A. Failure to return for HIV test results among persons at high risk for HIV in- fection. Results from a multistate interview project. $J$ Acquir Immune Defic Syndr 2004; 35:511-518.

3. Mkwanazi NB, Patel D, Newell M, Rollins NC, Coutsoudis A, Coovadia HM, Bland RM. Rapid testing may not improve uptake of HIV testing and same day results in a rural South African community: A cohort study of 12,000 Women. PLoS ONE 2008; 3(10): e3501. PubMed

4. Centers for Disease Control and Prevention: HIV counseling and testing using rapid tests United States, 1995.

5. Greenwald JL, Burstein GR, Pincus J, Branson B: A rapid review of rapid HIV antibody tests. Curr Infect Dis Rep 2006; 8:125-131.

6. Cohen MH, Olszewski Y, Branson B, Robey M, Love F, Jamieson DJ, Bulterys M. Using point-of-care testing to make rapid HIV-1 tests in labor really rapid. AIDS 2003; 17:2121-2124. PubMed

7. Moodley D, Moodley P, Ndabandaba T, Esterhuizen T. Reliability of HIV rapid tests is user dependent. $S A M J$ 2008; 98(9):0707-709.

8. Pronyk PM, Kim JC, Makhubele MB, Hargreaves JR, Mohlala R, Hausler HP: Introduction of voluntary counselling and rapid testing for HIV in rural South Africa: from theory to practice. AIDS Care 2002;14:859-865. PubMed

9. World Health Organization Regional Office for Africa, Centers for Disease Control and Prevention, and Association of Public Health Laboratories.Guidelines for Appropriate Evaluations of HIV Testing Technologies in Africa. Centers for Disease Control and Prevention, Atlanta, GA, 2003.

10. Kanal K, Chou TL, Sovann L, Morikawa Y, Yumi M, Kazuhiro K. Evaluation of the proficiency of trained non-laboratory health staffs and laboratory technicians using a rapid and simple HIV antibody test. AIDS Res Ther 2005;2:5.

11. National Department of Health. South African National HIV Counselling and Testing (HCT) Policy Guidelines. Pretoria, Department of Health, 2010.

12. National Department of Health SA. HIV and AIDS and STI National Strategic Plan for South Africa 20072011. Pretoria, Department of Health, 2007.

13. Yao K, Wafula W, Bile EC, Cheignsong R, Howard S, Demby A, Nkengasong J. Ensuring the quality of HIV rapid testing in resource-poor countries using a systematic approach to training. Am J Clin Pathol 2010; 134:568-572. 14. Shanks L, Klarkowski D, O'Brien DP. False Positive 
HIV Diagnoses in Resource Limited Settings: Operational Lessons Learned for HIV Programmes. PLOS ONE 2013;8(3): e59906. PubMed

15. Klarkowski DB, Wazome JM, Lokuge KM, Shanks L, Mills CF, O'Brein DP. The Evaluation of a Rapid In Situ HIV Confirmation Test in a Programme with a high Failure Rate of the WHO HIV Two-Test Diagnostic Algorithm. PLoS ONE 2009;4(2): e4351. PubMed

16. World Health Organisation. Rapid HIV tests: guidelines for use in HIV testing and counselling services in resource-constrained settings WHO Press, World Health Organization, 20 Avenue Appia, 1211 Geneva 27, Switzerland. Geneva: WHO, 2004.

17. World Health Organization. Guidelines for assuring the accuracy and reliability of HIV rapid testing. Geneva, Switzerland: World Health Organization, 2005.
18. Shott JP, Galiwango RM, Reynolds SJ. A quality management approach to Implementing point-of-care technologies for HIV diagnosis and monitoring in Sub-Saharan Africa. J Trop Med 2012; 2012:651927.

19. Parekh BS, Ellenberger D, Westerman L, Yang C, Nkengasong JN. Human immunodeficiency virus (HIV) infections. In: Unemo M (Ed.) Laboratory diagnosis of sexually transmitted infections, including human immunodeficiency virus (pp. 155-166). Geneva, Switzerland: World Health Organization, 2013.

20. National Department of Health, South Africa. Guidelines for assuring the accuracy and reliability of HIV rapid testing: Applying a quality systems approach. Pretoria: Department of Health, 2009.

21. Dewsnap CH, McOwan A. A review of HIV point of care tests. Int J STD AIDS 2006;17:357-9. PubMed 